\title{
Winter Wheat Quality Inspection and Regionalization Based on NIR Network and Remote Sensing
}

\author{
Xiaodong Yang*, WenJiang Huang, Cunjun Li, Xingang Xu, and Hao Yang \\ National Engineering Research Center for Information Technology in Agriculture, \\ Beijing 100097, China \\ \{yangxd, huangwj, licj, xuxg, yangh\} @nercita.org.cn
}

\begin{abstract}
In the crown of the year, inspection of wheat quality fast and accurate is very important for all of grain enterprises, farmers and governments. Governments would like to construct a fair and equitable market for grain transaction with explicit grain quality standard. Farmers would like to sell their high-quality grain at a high unit price for they have paid more attention and investment. Enterprises also would like to purchase high-quality grain with higher unit price for it can bring more profits. At the same time, generating regionalization map of wheat quality accuracy in time is very important on the grain enterprises' purchase strategy formulating and purchase region choosing. The authors collected 1200 NIR samples in 240 points (in other words, 5 samples per point ) in 12 counties in the main wheat producing areas in China (Hebei, Henan, Jiangsu and Shandong), then analysis these samples by both GIS spatial interpolation method and RS inverse method. In contrast, RS inverse method can simulate the quality parameter more accuracy than GIS spatial interpolation method. In conclusion, RS inverse method is preferable to generate quality regionalization map with NIR network samples.
\end{abstract}

Keywords: Winter Wheat; Quality Inspection; Quality Regionalization; NIR Network; Remote Sensing.

\section{Introduction}

Near infrared (NIR) technology has been widely used in quality inspection in China and now only a single NIR instrument is usually used to inspect the sample which can evidently improve the efficiency of inspection [1]. However, a single NIR instrument lacks the universality and uniformity because its calibration model is the default model of producers or the model being generated according local samples. In this situation, all the instruments not in network only can inspect samples in the local Lab with higher reliability and higher accuracy; while the inspection results between different instruments may be have large difference (known as difference between instruments) [2].

\footnotetext{
* Corresponding author.
}

This work was supported by Non-profit Agriculture Research Special Fund support by Ministry of Agriculture of the People's Republic of China (200903010 \& 201003039). 
Grain Quality Inspection NIR Network can make up the gap to achieve the consistency and credibility of results between instruments by transferring calibration model to Client-Instrument from Host-Instrument through the server in Network Management Center. The stable calibration model provided by the unified analysis network can ensure all devices within the network producing the inspection results having the same accuracy and stability and make all devices not being influenced by the difference between the sample sources and the test environment. It's the important technology base of Grain Quality Analysis in large scale region to implement the inspection results of any instrument in the NIR network having the same accuracy and reliability with integrating with GIS or RS [3].

Remote sensing technology is widely used in crop growth monitoring, pests monitoring and quality monitoring since it has a natural advantage in the analysis of distribution and change of land cover on earth [4]. But we can't get the full crop growth information from RS data directly since RS data is comprehensive, namely the pixel value in the image is a comprehensive reflection of the features located in the certain region and the information in the image cannot completely be interpreted to the corps growth information [5].

From above can be concluded that NIR Network technology can inspect grain quality accurately, rapidly and reliably while RS monitoring technology can expand the accurate information of the earth's surface from point to plane by a reverse model. So we can generate the quality regionalization map for crop enterprise and government by combining these two technologies. The enterprise can purchase the appropriate and necessary crop with higher price according this map.

\section{Material and Methods}

\subsection{The Structure of NIR Network}

NIR network technology has been widely used in Europe and America. Now there more than 30 worldwide NIR grain quality analysis networks which are composed by nearly ten thousand NIR instrument running in the United States, Canada, Germany, France and Australia, etc [6]. More and more quality analysis of crop trading in Europe and the United States were inspected by NIR network. NIR Analysis technology has been certificated by a number of international organizations and national official, including the United States, Canada, Germany, France, Australia, etc [7].

The first NIR network for crop quality analysis in china was established by NERCITA consisting of a number of universities, research institutes and crop enterprises in Heilongjiang, Jilin, Hebei, Shandong, Jiangsu and Hunan [8].

NIR grain quality monitoring network is composed by Network Steering Committee, Calibration Center, Network Management Center and Client Users (As shown in Fig.1). The functions of every component are described as below:

Network Steering Committee's task is to determine to use the new calibration or adjust the existed calibration based on the statistics from the calibration center.

Calibration Center's task is to develop the application model for all instruments in network.

Network Management Center's task is to manage and maintain the network.

Client Instrument is the end-user of the network. 


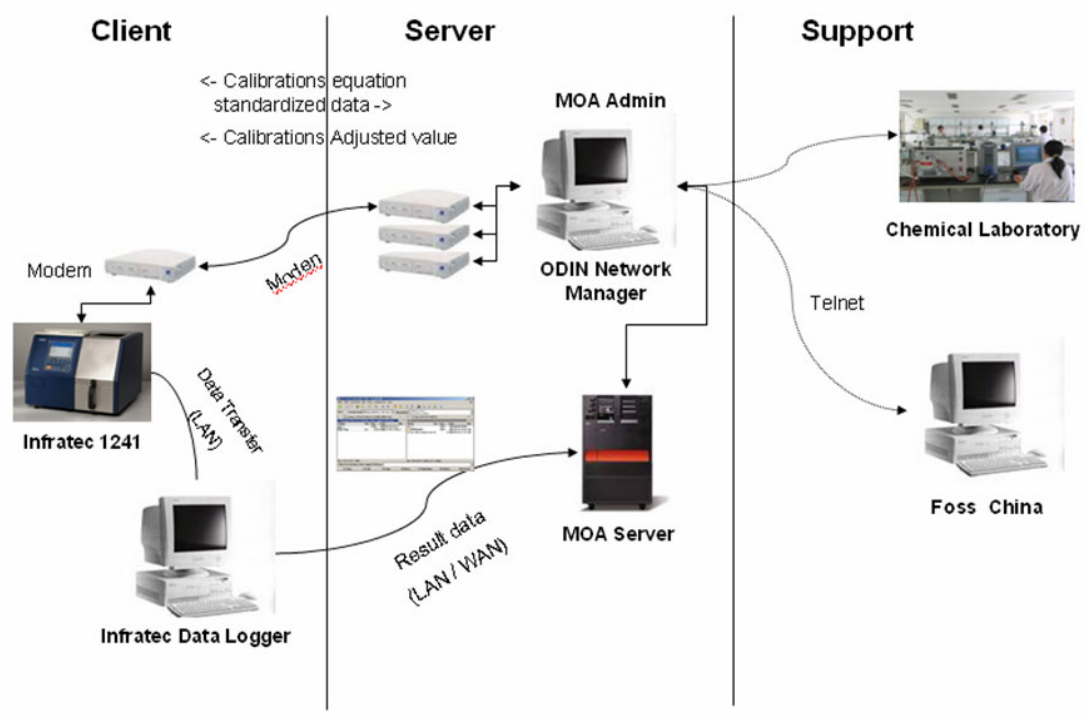

Fig. 1. The structure of the NIR network in NERCITA

\subsection{Calibration Transferring and Instruction Standardization}

Calibration transferring and instruction standardization can eliminate the model difference generated by the host and client instructions manufacturing tolerances [9]. The common method is to analyze a same group samples by host and client instruments, then to adjust the slope and intercept of calibration model of client instruments. The authors adjusted 7 instruments in the NIR network for Proton, moisture and wet gluten of winter wheat, the results are shown in Table $1 \sim$ Table 3 , the instruments in the network are accuracy and consistent.

Table 1. The accuracy of calibration transfer and model adjustment for Protein

\begin{tabular}{ccccccc}
\hline Instrument & RMSEP & Bias & Slope & Intercept & Correlation \\
\cline { 1 - 2 } Hebei-1 & 0.05 & -0.03 & 0.98 & 0.40 & 1.00 \\
Henan-1 & 0.07 & 0.00 & 0.97 & 0.42 & 1.00 \\
Henan-2 & 0.10 & -0.08 & 0.98 & 0.45 & 1.00 \\
Jiangsu-1 & 0.03 & -0.01 & 0.97 & 0.42 & 1.00 \\
Shandong-1 & 0.11 & -0.11 & 1.00 & 0.04 & 1.00 \\
Shandong-2 & 0.07 & 0.01 & 0.97 & 0.37 & 1.00 \\
Jiangsu-2 & 0.04 & -0.02 & 0.96 & 0.56 & 1.00 \\
\hline
\end{tabular}


Table 2. The accuracy of calibration transfer and model adjustment for Moisture

\begin{tabular}{cccccc}
\hline \hline Instrument & RMSEP & Bias & Slope & Intercept & $\begin{array}{c}\text { Correla- } \\
\text { tion }\end{array}$ \\
\hline Hebei-1 & 0.03 & -0.02 & 0.97 & 0.36 & 1.00 \\
Henan-1 & 0.14 & -0.14 & 0.92 & 1.04 & 0.99 \\
Henan-2 & 0.18 & -0.18 & 0.93 & 0.97 & 0.99 \\
Jiangsu-1 & 0.04 & -0.02 & 0.92 & 0.98 & 0.99 \\
Shandong-1 & 0.05 & 0.04 & 0.94 & 0.63 & 0.99 \\
Shandong-2 & 0.02 & -0.02 & 0.99 & 0.10 & 1.00 \\
Jiangsu-2 & 0.04 & -0.03 & 0.93 & 0.81 & 1.00 \\
\hline
\end{tabular}

Table 3. The accuracy of calibration transfer and model adjustment for Wet Gluten

\begin{tabular}{cccccc}
\hline Instrument & RMSEP & Bias & Slope & Intercept & $\begin{array}{c}\text { Correla- } \\
\text { tion }\end{array}$ \\
\hline Hebei-1 & 0.24 & 0.06 & 1.16 & -5.43 & 1.00 \\
Henan-1 & 0.93 & 0.53 & 0.69 & 9.73 & 0.98 \\
Henan-2 & 0.49 & 0.40 & 0.96 & 0.77 & 0.99 \\
Jiangsu-1 & 0.59 & 0.32 & 0.78 & 6.94 & 0.99 \\
Shandong-1 & 0.41 & -0.07 & 1.29 & -9.42 & 0.99 \\
Shandong-2 & 0.16 & 0.04 & 1.03 & -1.17 & 1.00 \\
Jiangsu-2 & 0.38 & 0.33 & 0.95 & 1.27 & 0.99 \\
\hline
\end{tabular}

\subsection{Samples Collecting}

The samples were collected from 12 counties in Henan, Hebei, Shandong and Jiangsu, the main winter wheat belt of China. For every sample point, the authors recorded detail information including GPS location, wheat variety name, sample sizes and sampling time. On every site, the authors collected 5 samples- 2 for the NIR network detection, 2 for chemical contrast and 1 for backup. The factors of detection included protein content, moisture content and wet gluten content.

Firstly, the Infratec Data Logger - a special soft for data collecting developed by FOSS - collected the inspection data from instrument to PC which connected with the Instrument by intranet. Then these data were uploaded to the server in network management center by a FTP synchronization software tools. 


\subsection{Methods}

\subsubsection{GIS Interpolation}

In our research, an optimal space interpolation method-Kriging method- was used to analysis the moisture, protein and wet gluten in wheat for getting the spatial distribution map of the wheat quality class interpolation. The map can reflect the quality class of wheat.

The fundamental principles of Kriging method is similar to least squares method and its essence is to consider not only the general trend changes (statistical features), but also the related changes and the random variations on the sample surface [10]. The general trend changes, the related changes and the random variations are respectively named the structured item, the relevant item and the random noise (as shown in Fig.2) [11]. The usual fitting function equation is as follows:

$$
f(x, y)=f_{1}(x, y)+f_{2}(x, y)+C
$$

Where $f_{1}(x, y)$ is the structured item, $f_{2}(x, y)$ is the relevant item and $C$ is the random noise.

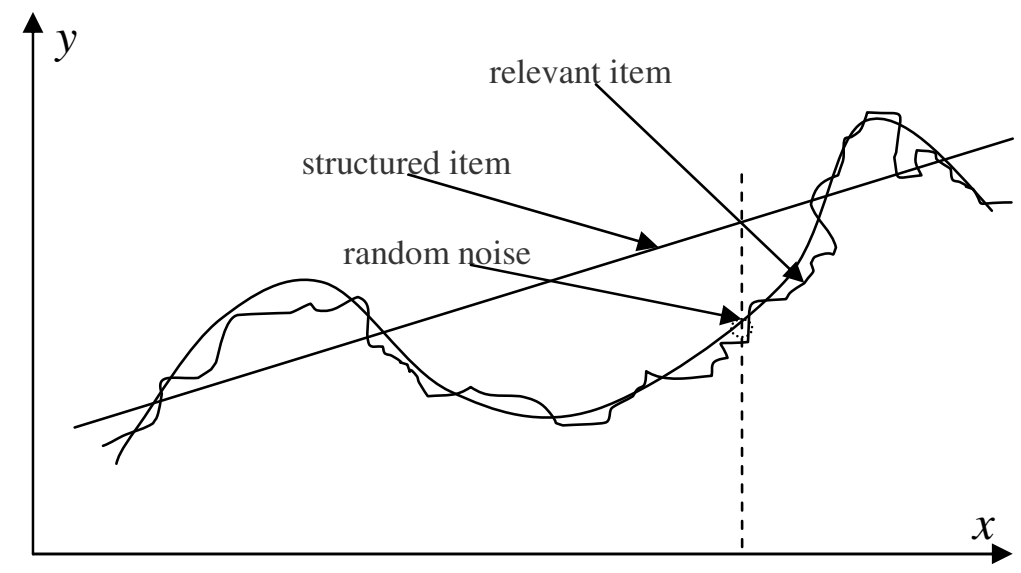

Fig. 2. The principle of Kriging interpolation method

\subsubsection{RS Inverse Model}

The basis method of RS inverse is to set up a correlation model between the RS information and the real system on earth [12]. Firstly the linear or non-linear relationship between the crop growth state index and the single phase or multi-temporal spectral vegetation index (VI) is analyzed; then based on the relevant relations the statistic model, including the single variable and the multivariate statistical models, is set up. In addition, there is the RS inverse method based on the priori knowledge, which mainly uses the Bayesian statistical method. Our research adopts the single variable statistical models and the formula of correlation coefficient between the crop growth state index and the vegetation index is 


$$
R_{x y}=\frac{\sum_{i=1}^{n}\left(x_{i}-\bar{x}\right)\left(y_{i}-\bar{y}\right)}{\sqrt{\sum_{i=1}^{n}\left(x_{i}-\bar{x}\right)^{2}} \cdot \sqrt{\sum_{i=1}^{n}\left(y_{i}-\bar{y}\right)^{2}}}
$$

Where Rxy is correlation coefficient value, $\mathrm{Xi}$ is the observed value of the crop growth state index of No.i sample point, Yi is the value of spectral vegetation index of No.i sample point, $x$ is the average value of the observed value of the crop growth state index of all sample points and $\bar{y}$ is the average value of spectral vegetation index of all sample points.

\section{Results and Discussion}

\subsection{Accuracy Analysis of NIR Network}

The authors selected 240 sample points in 12 counties in the main wheat producing areas in China (Hebei, Henan, Jiangsu and Shandong, as shown in Figure 2.) in 2009. In every point, the authors got 5 samples, 2 for the near-infrared detection, 2 for chemical assay, the test results averaged. Comparison of detection of one protein results shown in Fig. 3:

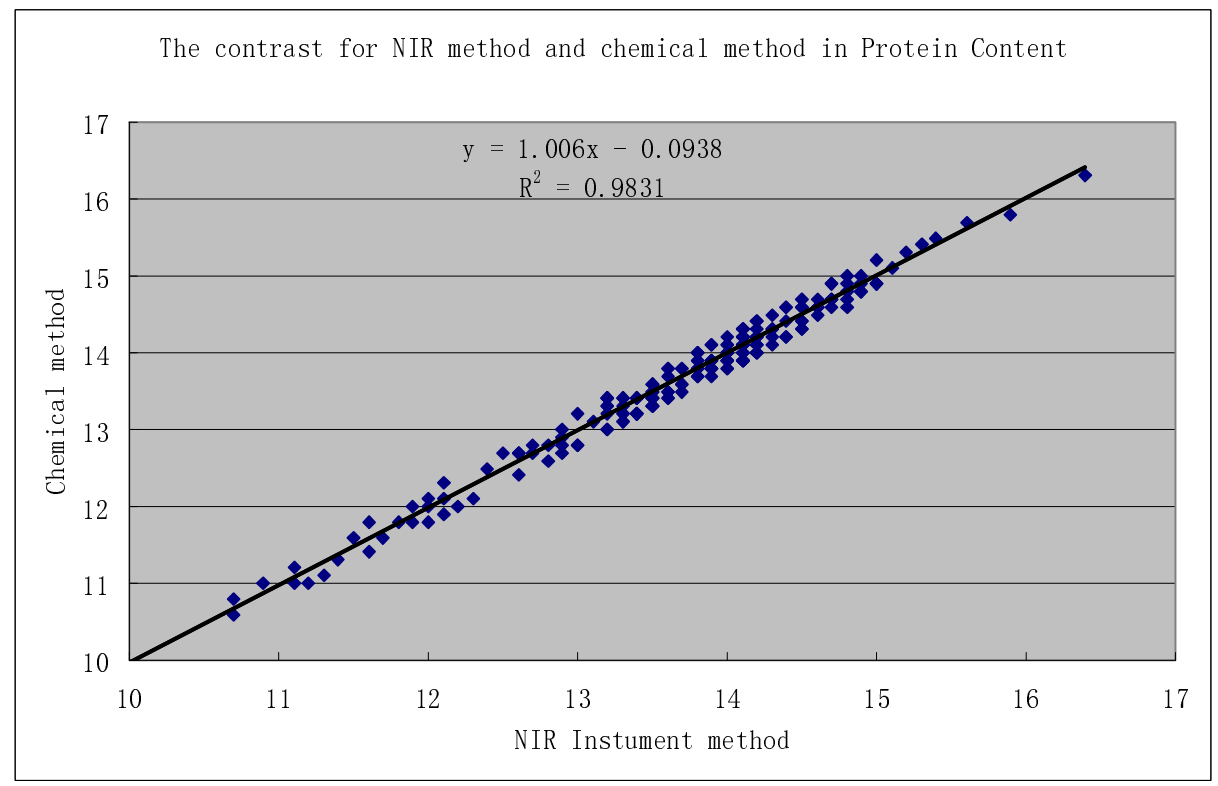

Fig. 3. The accuracy of NIR network contrast to Chemical method 


\subsection{Winter Wheat Quality Regionalization by Integration with GIS Interpolation}

GIS technology is used in spatial analysis field widely. The GIS spatial interpolation map of samples points as shown in Fig. 4.

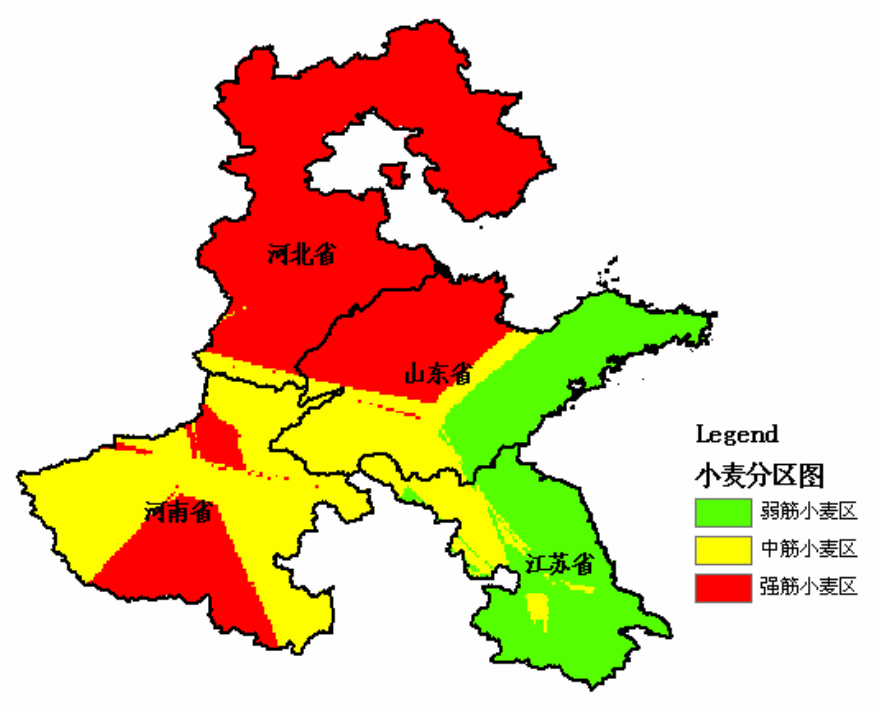

Fig. 4. The winter wheat quality thematic map generate by GIS spatial interpolation

Because the collection of samples are limited by regions, labors, date and others, it is difficult to collect samples at large-scale and on certain percentage. The thematic map generate by GIS technology is always not very accuracy and reliable for the lack of enough sample points.

\subsection{Winter Wheat Quality Regionalization by Integration with RS Inverse Model}

RS image is taken in a moment, so the information represented by its pixel value is steady and sequential. Some vegetation indexes (VI) generated by RS image can reflect the quality information of covered crop so the inverse model having a high correlation can derive the regional quality thematic map reliable.

The authors chose randomly 115 of the 240 sample points to build inverse model and the other 125 points to verify the model. The details are shown in Table 4 . The inverse thematic map is shown in Fig. 5.

Table 4. Detail info of sample points assign and accuracy contrast between GIS method and RS method

\begin{tabular}{rcccc}
\hline & Hebei & Henan & Jiangsu & Shandong \\
\hline Points for build Mode1 & 40 & 40 & 35 & 0 \\
Points for verification & 35 & 30 & 20 & 40 \\
standard deviation of GIS & 0.134 & 0.117 & 0.122 & 0.178 \\
standard deviation of RS & 0.048 & 0.055 & 0.054 & 0.102 \\
\hline
\end{tabular}




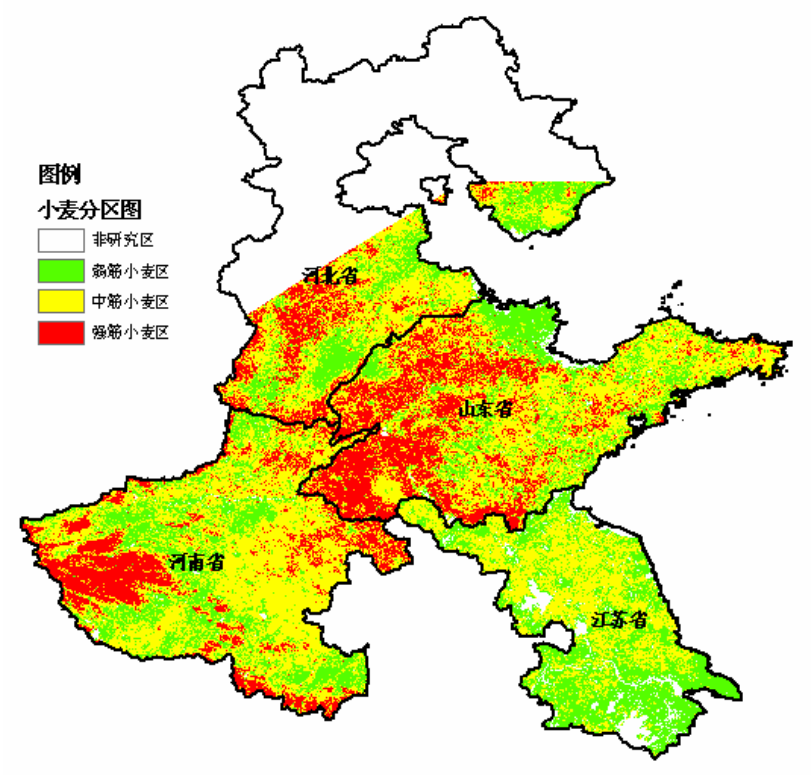

Fig. 5. The winter wheat quality thematic map generate by RS inverse model

\section{Conclusion}

By above comparison, the Quality Regionalization map generated by RS inverse model is more accuracy and reliable than by GIS spatial interpolation map. So we can conclude that Integration RS monitoring technology with NIR network to generate Quality Regionalization map is valuable for crop enterprise on making purchase policy and bringing interests to enterprises, farmers and governments at the same time.

\section{References}

1. Yan, Y., Zhao, L., Han, D., Yang, S.: Elements and application of Near-Infrared Spectra Analysis. China Light Industry Press, Beijing (2005)

2. Wang, Y., Veltkamp, D.J., Kowalski, B.R.: Analytical Chemistry 63, 2750 (1991)

3. Lu, W.-.z.: Modern Near Infrared Spectroscopy Analytical Technology, 2nd edn. China Petrochemical Press, Beijing (2007)

4. Humburg, D.S., Stange, K.W., Robert, P.C., et al.: Spectral properties of sugar beets related to sugar content and quality. In: Proceedings of the Fourth International Conference on Precision Agriculture. St. Paul, M Minnesota, USA, July19- 22, (1998) Part A and Part B, pp. 1593-1602 (1999)

5. Wang, J.H., Huang, W.J., Zhao, C.J.: Estimation of leaf biochemical components and grain quality indicators of winter $\mathrm{w}$ heat from spectral reflectance. Journal of Remote Sensing 7(4), 277-284 (2003) 
6. Wei, Y.M.: Corn Quality and Food Processing —Wheat Grain Quality and Food Processing. China Agricultural Science and Technology Press, Beijing (2005) (in Chinese)

7. Zhang, X., Liu, L.Y., Zhao, C.J., Zhang, B.: Estimating wheat nitrogen concentration with high spectral resolution image. Journal of Remote Sensing 7(3), 176-181 (2003) (in Chinese)

8. Wang, J.H., Wang, Z.J., Huang, W.J., Ma, Z.H., Liu, L.Y., Zhao, C.J.: The vertical distribution characteristic and pectral response of canopy nitrogen in different layer of winter wheat. Journal of Remote Sensing 8(4), 309-316 (2004) (in Chinese)

9. Zubillaga, M., Urricariet, S.: Assessment of nitrogen status in wheat using aerial photography. Communications in Soil Science and Plant Analysis 36(13-14), 1787-1798 (2005)

10. Wang, Z.J., Wang, J.H., Liu, L.Y., Huang, W.J., Zhao, C.J., Wang, C.Z.: Prediction of grain protein content in winter wheat (Tritium aestivum L.) using plant pigment ratio (PPR). Field Crops Research 90(2-3), 311-321 (2004)

11. Wu, L.X., Shi, W.Z.: Principles and Algorithms of GIS. Science Press, Beijing (2003) (in Chinese)

12. Li, W.G., Wang, J.H., Zhao, C.J., Liu, L.Y., Guo, W.S.: Study on monitoring starch content in winter wheat grain using Land-Sat TM image. Journal of Yunnan Agricultural University 22(3), 365-369 (2007) (in Chinese) 